\title{
Anatomic variability of groin innervation
}

\author{
P. Bachul1, ${ }^{1}$, K.A. Tomaszewski ${ }^{1}$, E.K. Kmiotek ${ }^{1}$, M. Kratochwil ${ }^{2}$, R. Solecki ${ }^{2}$, J.A. Walocha ${ }^{1}$ \\ ${ }^{1}$ Department of Anatomy, Jagiellonian University Medical College, Krakow, Poland \\ 2Department of General, Oncological and Gastroenterological Surgery, Jagiellonian University Medical College, \\ Krakow, Poland
}

[Received 6 April 2013; Accepted 9 May 2013]

Inguinal hernia repairs are very common yet fairly complex surgical procedures. Variations in the anatomical course of the inguinal nerves require that diligence is taken in their proper recognition. Inadvertent surgical injury to these nerves is associated with long term postoperative pain and complications. The aim of the present study was to highlight the complexity and variation in the innervation of the inguinal region in order to increase proper nerve identification during surgical interventions. Bilateral dissection of the inguinal and posterior abdominal regions in one human male cadaver revealed an atypical anatomic topography of the groin innervation. This unusual case was observed at the Jagiellonian University Anatomy Department during routine cadaveric preparations. The left ilioinguinal nerve was absent. The left genital branch of the genitofemoral nerve arose higher than expected from the lumbar plexus and supplied the groin region, which is typically innervated by the ilioinguinal nerve. Furthermore, the left lateral cutaneous femoral nerve and the right genital branch of the genitofemoral nerve also followed uncharacteristic courses. Awareness of topographical nerve variations during inguinal hernia repair will help surgeons identify and preserve important nerves, thus decreasing the incidence of chronic postoperative pain. (Folia Morphol 2013; 72, 3: 267-270)

Key words: inguinal hernia, groin, variability, ilioinguinal nerve, genitofemoral nerve, cutaneous femoral nerve

\section{INTRODUCTION}

Inguinal hernia repairs are among the most commonly performed procedures by general surgeons. Each year, there are over 800,000 such surgeries completed in the US [8]. In Europe, the number of repairs is also high, with 200,000 per year in Germany and 60,000 per year in Poland [9]. The rich innervation of the inguinal region makes this area particularly sensitive during lower abdominal surgery. Variation in the anatomical course of the inguinal nerves further complicates this already demanding procedure as nerves may be inadvertently compressed or sectioned. Injury to the innervation of this area can lead to postoperative pain $[6,7]$. For instance, in 49 out of 110 hernia repairs reported by Al-Dabbagh [1], the atypical superficial placement of the ilioinguinal nerve left it particularly vulnerable to transection in the early stages of the operation. The nerve was found either emerging through the fibres of the external oblique muscle, proximal to the superficial inguinal ring or running parallel and in proximity to the latter [1]. Such placement increases the risk of it being severed when the external oblique aponeurosis and superficial inguinal ring are split [5]. These findings highlight the importance of considering atypical anatomic innervation during lower abdominal surgery in preventing nerve injury.

In the present study, the anatomical variations in the course of the genitofemoral, lateral cutaneous 
femoral, ilioinguinal, and iliohypogastric nerves are described. Knowledge of the course of these nerves in the inguinal region, and an understanding of variations seen in their topography, can decrease the incidence of postoperative complications and adverse effects.

\section{CASE REPORT}

An elderly male cadaver, age 75 , fixed in a $10 \%$ solution of formaldehyde, was carefully prepared and examined. The inguinal region of the cadaver was dissected bilaterally and the course of the inguinal nerves was followed. Inspection of the inguinal region and posterior abdominal cavity, on the left side of the specimen, showed that the left ilioinguinal nerve was absent. Furthermore, the left genital branch of the genitofemoral nerve coursed atypically. It arose much higher from the lumbar plexus than would be expected and it did not appear to have an evident place of origin (Fig. 1). As the general purpose of the dissection was to explore the anatomy of the inguinal region, and not to explore the entire course of the genitofemoral nerve, further dissection to locate its origin was not conducted. The nerve continued downward in the direction of the inguinal canal, pierced the lateral border of the psoas major muscle, and continued to run anterior to it. In the inguinal canal the genital branch passed just below the spermatic cord, within the cremasteric fascia, and innervated the cremasteric muscle. Next, it left the inguinal canal through the superficial inguinal ring, as a sensory branch, and provided innervation to the medial thigh. This region of the groin is typically supplied by the ilioinguinal nerve. Above the spermatic cord, the left iliohypogastric nerve was identified within its normal topography (Fig. 2). Equally, the left femoral branch of the genitofemoral nerve followed the typical anatomical course below the inguinal ligament (Fig. 3). The left lateral cutaneous femoral nerve, in relation to the anterior superior iliac spine, coursed considerably lower than expected (Fig. 1). Instead of traveling at the level of the spine, it passed several centimetres below it.

In the right inguinal region of the cadaver, the course of the genital branch of the genitofemoral nerve was identified. It emerged at the medial margin of the psoas major muscle and coursed along the right common and external iliac arteries, towards the posterior wall of the inguinal canal. There, in the area

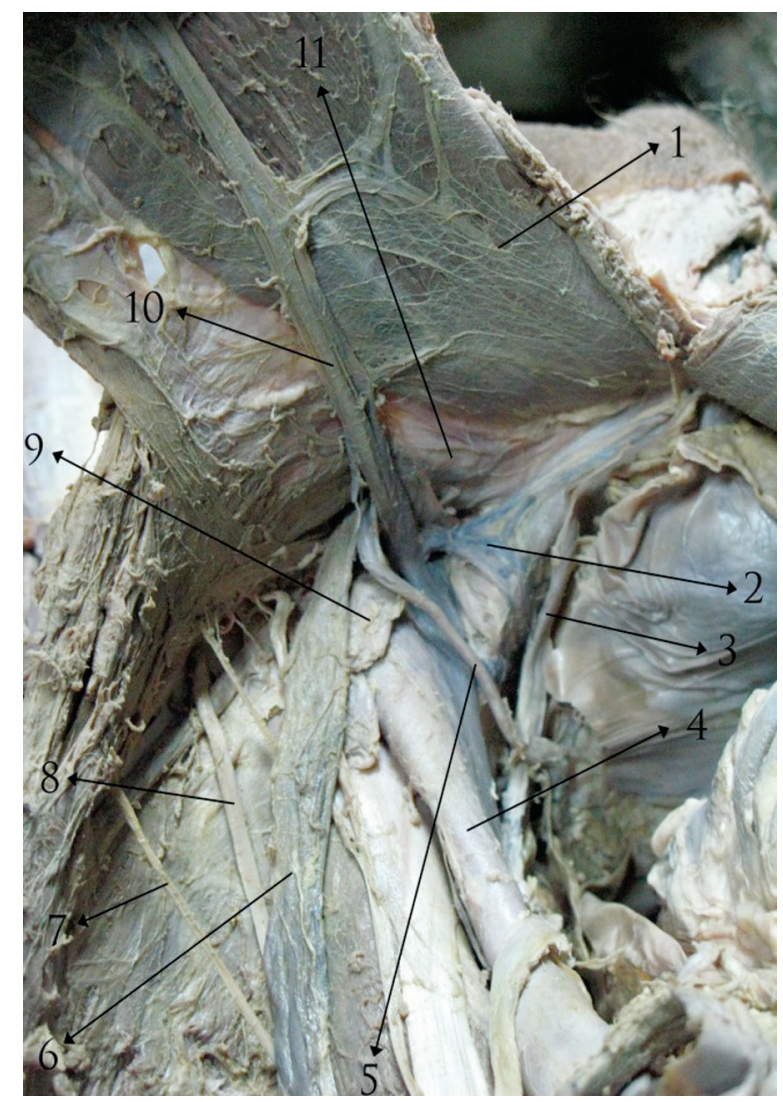

Figure 1. Left inguinal region; 1 - rectal abdominis muscle; 2 venous type of "corona mortis"; 3 - medial umbilical ligament; 4 - external iliac artery; 5 - vas deferens; 6 - testicular vessels; 7 - lateral cutaneous femoral nerve; 8 - femoral branch of the genitofemoral nerve; 9 - lymphatic node; 10 - inferior epigastric vessels; 11 - transversalis fascia.

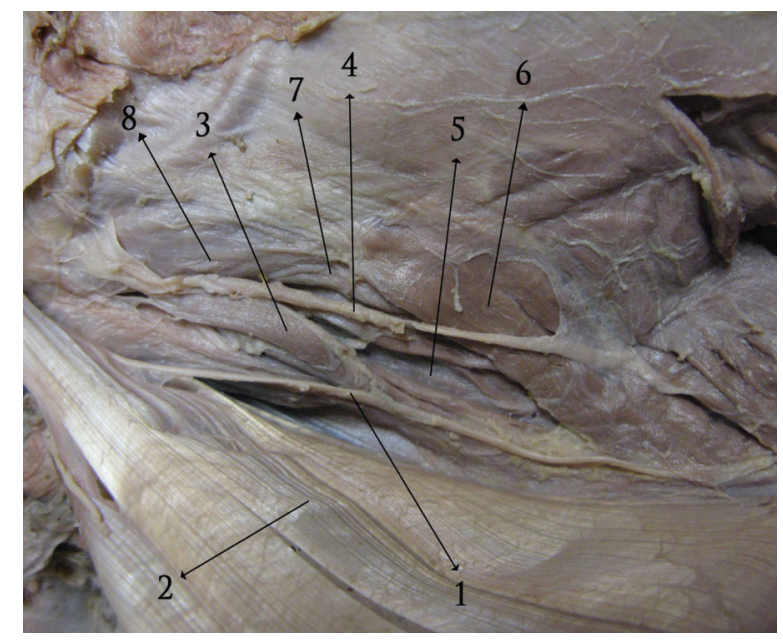

Figure 2. Left inguinal region; 1 - ramus genitalis nervi genitofemoralis; 2 - aponeurosis musculi obliqui abdominis externi; 3 musculus cremaster; 4 - iliohypogastric nerve; 5 - transversalis fascia; 6 - musculus obliqus internus abdominis; 7 - musculus transversus abdominis; 8 - falx inguinalis (conjoint tendon). 


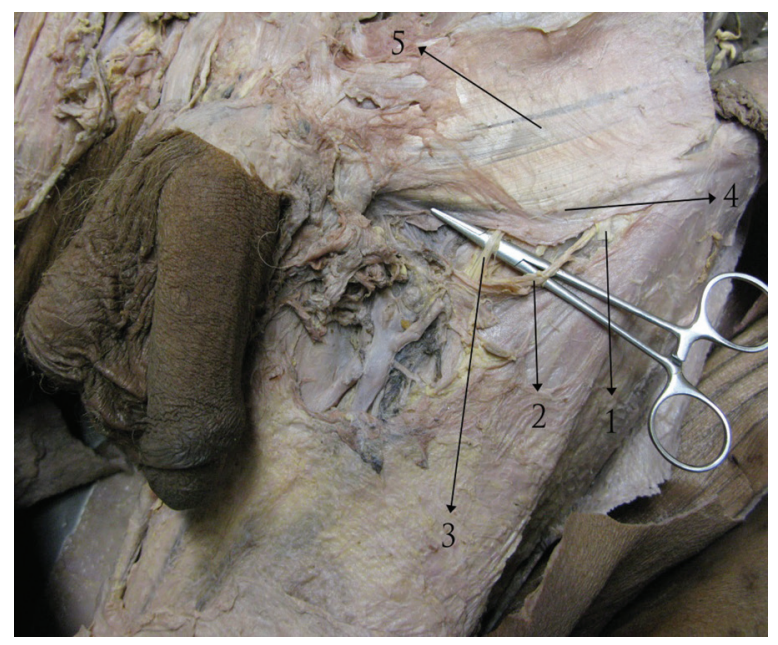

Figure 3. Left inguinal region; 1 - nervus cutaneus femoris lateralis; 2 - vasa circumflexa ilium superficiales; 3 - ramus femoralis nervi genitofemoralis; 4 - ligamentum inguinale; 5 - aponeurosis musculi obliqui abdominis externi.

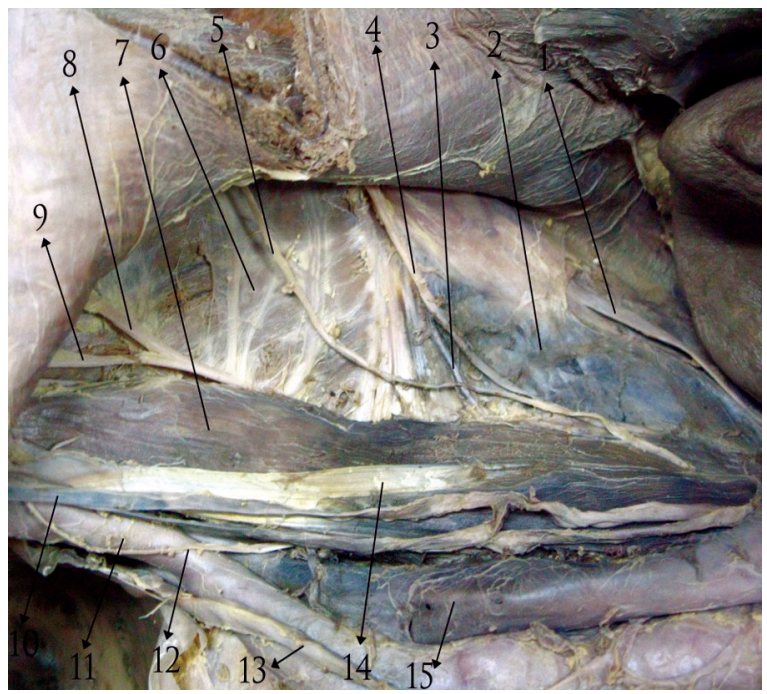

Figure 4. Right inguinal region; 1 - nervus subcostalis; 2 musculus quadratus lumborum; 3 - vena lumbalis; 4 - nervus iliohypogastricus; 5 - nervus ilioinguinalis; 6 - musculus iliacus; 7 - musculus psoas major; 8 - nervus cutaneus femoris lateralis; 9 - ramus femoralis nervi genitofemoralis; 10 - vena testicularis; 11 - arteria iliaca externa; 12 - ramus genitalis nervi genitofemoralis; 13 - ureter dexter; 14 - tendo musculi psoas minor; 15 - vena cava inferior.

of Hasselbach's triangle, it pierced the transversalis fascia and entered the canal (Fig. 4). Resection of the aponeurosis of the external oblique muscle revealed that the genital branch of the genitofemoral nerve exited the inguinal canal through the superficial

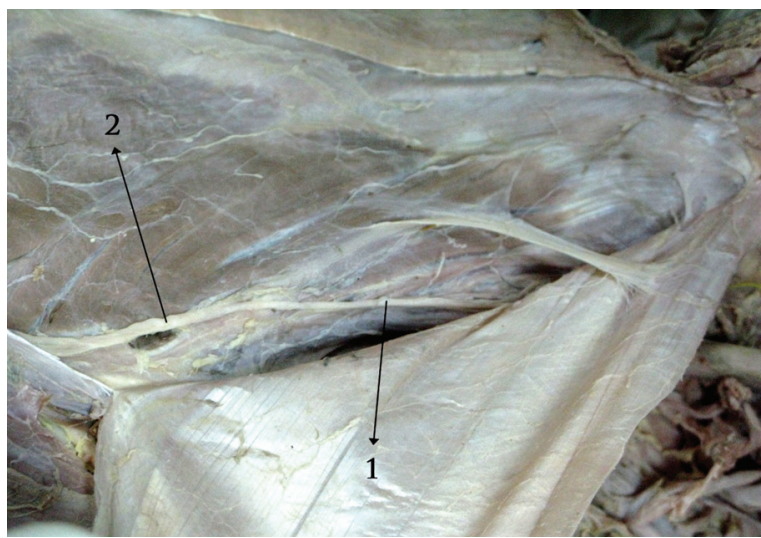

Figure 5. Right inguinal region; 1 - ramus genitalis nervi genitofemoralis - one can see the anastomosis between this ramus and the ilioinguinalis nerve; 2 - nervus ilioinguinalis.

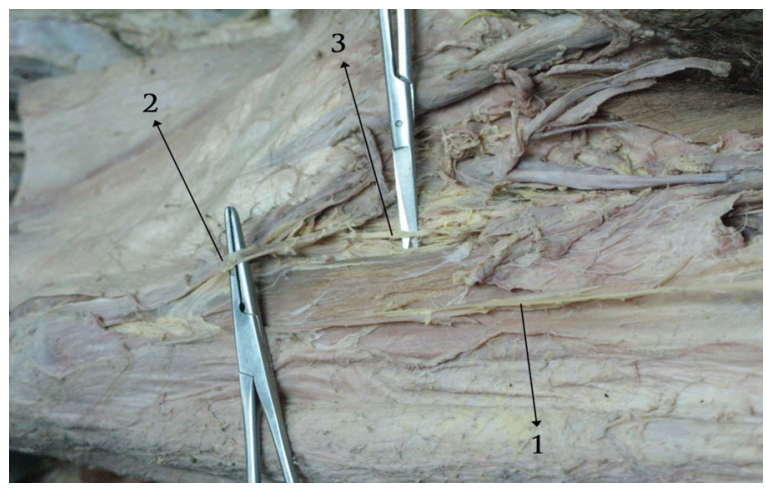

Figure 6. Right inguinal region; 1 - nervus cutaneus femoris lateralis; 2 - vasa circumflexa ilium superficiale; 3 - ramus femoralis nervi genitofemoralis.

inguinal ring, together with ilioinguinal nerve, and provided sensory innervation to the right groin area (Fig. 5). The courses of the femoral branch of the genitofemoral, the iliohypogastric, and lateral cutaneous femoral nerves were topographically normal (Figs. 4, 6).

\section{DISCUSSION}

Lower quadrant surgeries, such as hernia repairs, may cause injury to the inguinal nerves. Damage to the inguinal nerves has been associated with long term postoperative groin pain $[2,3,7]$. The highest level of self-reported postoperative pain, at 3 months, has been found to be correlated with non-identification of the ilioinguinal nerve [7]. Fortelny and Petter Puchner [3] estimate the incidence of postoperative 
groin pain to be around $31 \%$, with $6 \%$ of respondents reporting interference with daily activities [4]. As such, detailed knowledge of the variability of the inguinal innervation is critical for early identification and sparing of the nerves. The present study describes the variability in the course of the genitofemoral, lateral cutaneous femoral, ilioinguinal, and iliohypogastric nerves as seen in an elderly male cadaver.

The inguinal nerves are often found to follow variable patterns of distribution $[1,5,6]$. In a recent study of patients undergoing hernia repair, Al-Dabbagh [1] found that the course of the ilioinguinal and iliohypogastric nerves was only consistent with anatomic texts in $41.8 \%$ of cases. Furthermore one or both of these nerves was found to be absent in $7.3 \%$ of cases [5]. In the current cadaveric dissection, an atypical pattern of inguinal nerve branches was found. The left genital branch arose higher than expected from the spine, the left lateral cutaneous femoral nerve coursed significantly below the superior anterior iliac spine, and the right genital branch of the genitofemoral nerve followed an uncharacteristic course. Lastly, the left ilioinguinal nerve was absent.

In light of the great variability of the course of the inguinal nerves, a single description of the topography of the inguinal innervation is insufficient to properly identify these nerves. Instead, surgeons must consider the various patterns of branching that can occur in the region, to avoid accidental nerve damage. Rab et al. [6] categorised the distribution patterns of the ilioinguinal and genitofemoral nerves into 4 different types: $A, B, C$, and D. The course of the left genital branch reported in this cadaveric dissection corresponds to the type A pattern. According to this classification, the skin of the pubis and ventral scrotum or labia is innervated only by the cutaneous component of the genital branch of the genitofemoral nerve [6]. This innervation was found in the dissected specimen. Such a conformation was reported to be the most common and was present in $43.7 \%$ of cadavers studied by Rab et al. [6].

The course of the right genitofemoral nerve, found in this present cadaver, corresponds to the more rare, type $D$ pattern $(7.8 \%$ of dissections), where the cutaneous components of the ilioinguinal and genitofemoral nerves together supply the skin of the groin region [6]. The innervation of the right and left inguinal regions, reported in the current male cadaver, are asymmetrical in their innervation pattern. Such a finding is supported by the observations of Rab et al. [6] who noted symmetric innervation in only $40.6 \%$ of dissected cadavers.

The major limitation of the present study is its "case report" nature. This manuscript reports the results of a single cadaveric dissection, and there is insufficient information to adequately define, in vivo, the roles that such nerve variations may have played during the patient's life. Nonetheless, the anatomic variations described in this case report suggest that, to properly understand the nerve distribution of the inguinal region, multiple patterns of innervation must be considered.

\section{CONCLUSIONS}

Concluding, a clear understanding of the anatomical locations of the inguinal nerves, during lower abdominal surgery, is pivotal in preventing nerve damage and subsequent complications. As highlighted by this paper, the topography of these nerves shows considerable variation. This must be taken into account when identifying these nerves. Knowledge of various atypical innervation patterns will allow surgeons to properly identify vital nerves and reduce the risk of postoperative groin pain and complications.

\section{REFERENCES}

1. Al-Dabbagh AKR (2002) Anatomical variations of the inguinal nerves and risks of injury in 110 hernia repairs. Surg Radiol Anat, 24: 102-107.

2. Campanelli G, Bertocchi V, Cavalli M, Bombini G, Tentorio T, Sfeclan C, Canziani M (2013) Surgical treatment of chronic pain after inguinal hernia repair. Hernia, [published online ahead of print March 22, 2013].

3. Fortelny RH, Petter Puchner AH (2010) A method for the reduction of chronic pain after tension-free repair of inguinal hernia: iliohypogastric neurectomy and subcutaneous transposition of the spermatic cord. Hernia, 14: 229-230.

4. Fränneby U, Sandblom G, Nordin P, Nyrén O, Gunnarsson U (2006) Risk factors for long-term pain after hernia surgery. Ann Surg, 244: 212-219.

5. Klaassen Z, Marshall E, Tubbs RS, Louis RG Jr, Wartmann CT, Loukas M (2011) Anatomy of the ilioinguinal and iliohypogastric nerves with observations of their spinal nerve contributions. Clin Anat, 24: 454-461.

6. Rab M, Ebmer J, Dellon AL (2001) Anatomic variability of the ilioinguinal and genitofemoral nerve: implications for the treatment of groin pain: Plast Reconstr Surg, 108: 1618-1623.

7. Smeds S, Lofstrom L, Eriksson O (2010) Influence of nerve identification and the resection of nerves 'at risk' on postoperative pain in open inguinal hernia repair. Hernia, 14: $265-270$.

8. Zendejas B, Ramirez $T$, Jones $T$, Kuchena A, Ali SM, Hernandez-Irizarry R, Lohse CM, Farley DR (2013) Incidence of inguinal hernia repairs in olmsted county, MN: a population-based study, Ann Surg, 257: 520-526.

9. Zieren J, Kupper R, Neuss PM, Muller JM (2003) Inguinal hernia: obligatory indication for elective surgery? Langensbeck's Arch Surg, 387: 417-420. 